\title{
Quality and texture analysis of biometric images compressed with second-generation wavelet transforms and SPIHT-Z encoder
}

\author{
Ahmed Bouida, Mohammed Beladgham, Abdesselam Bassou, Ismahane Benyahia \\ Information Processing and Telecommunication Laboratory (LTIT), \\ Faculty of Technology-University TAHRI Mohammed Bechar, Algeria
}

\begin{tabular}{l} 
Article Info \\
\hline Article history: \\
Received Dec 22, 2019 \\
Revised Mar 27, 2020 \\
Accepted Apr 8, 2020 \\
\hline
\end{tabular}

\section{Keywords:}

Biometric images

Glcm texture analysis

Image quality assessment

Quincunx wavelet transform

SPIHT encoder

SPIHT-z encoder

\begin{abstract}
In biometric systems, compression takes important place especially in order to reduce the size of the information stored or transmitted through the distributed biometric systems. It is also noted that the compression techniques induce loss of information in the compressed images that can affect the effectiveness of biometric systems. The main objective of our contribution is to examine the efficacy of the used method to offer an optimal compression quality in these kind of images without considerable distortion. In order to evaluate the efficacy of the compression process, we use two kinds of evaluation, full-reference image quality assessment and a new proposed textural quality analysis of the compressed images. In this paper, we use a second-generation wavelet transform to improve the compression study in biometric images. The basic idea of this algorithm is the quincunx wavelet transform coupled to a modified progressive encoder called SPIHT-Z encoding.
\end{abstract}

Copyright () 2020 Institute of Advanced Engineering and Science. All rights reserved.

\section{Corresponding Author:}

Ahmed Bouida,

Information Processing and Telecommunication Laboratory (LTIT),

University TAHRI Mohammed Bechar, Algeria.

Email: ahmedbouida@yahoo.fr

\section{INTRODUCTION}

The primary importance of compression techniques is to reduce the bit space required to store or transmit information of different types. Technically, the image compression algorithms are classed into two categories [1]. The first one is reversible compression or lossless methods. The reversible scheme make simple compression ratios, but allows exact recovery of the source image from the compressed version. The second type is irreversible compression or lossy methods, which does not allow exact recovery after the reconstruction of the compressed image, but it can reach higher compression ratios. The most normalized lossy compression technics use a transform method in order to decorrelate data information reducing the neighbors dependence. This transform phase is followed by a quantization and entropy coding phases. In the JPEG standard named as ISO/CEI 10918 [2], the basic used transform is the Discrete Cosine Transform (DCT). The JPEG2000 standard ISO/CEI 15444 [3], use the Discrete Wavelet Transform (DWT).

We note also that for solving problems of archiving and transmitting digital images in biometric domain, many compression methods are designed in goal to reduce the file size and to obtain a better image processing with a good image quality. The recent one is the ISO/IEC 19794 [4] standard for biometric data interchange formats, especially in fingerprint, face and iris data. In this standard, they define the lossy techniques used in data compression such as JPEG, WSQ and JPEG2000 applied in these biometric images. In some previous works, authors evaluate and verify the compression of the biometric images and their influence on biometric recognition systems [5-12]. The important goal of these studies is to study the impact of some compression algorithms like LZW, JPEG variants, WSQ, JPEG2000 and Wavelet packets (PWT) on 
the biometric images quality or the biometric recognition process using fingerprints, faces, iris and ear images. All these compression techniques are very responsive because they allow a considerable space saving. The only encountered problem is the degradation of image quality depending on the compression process and the required compression ratio. Generally and to perform the used compression algorithms, some evaluation methods are used to qualify the obtained images in compression process. Most of these techniques [13] are based on image quality index calculations by investigation in the pixel difference in images, visibility characteristic, contrast and structure features, a brain mechanism quality decision or using the human vision system model.

All of these methods support statistical analysis of spatial or spatio-frequency image features. In our investigation, the proposed verification is that to study the texture degradation in biometric images under the effect of the compression process. As compression algorithms, we use the second generation of Wavelet based on a quincunx structure algorithm to evaluate the compression quality on biometric images. This study compare the coupling of two progressive encoder; the SPIHT and recent modified SPIHT-Z encoders. For this aim and after presenting the overall work that study the evaluation of the compression effect on texture degradation in biometric images, this paper is organized into three parts: In the first part, we present the basic concepts used in the wavelet transform algorithm by evoking the second-generation scheme and the used progressive encoding techniques. To evaluate the quality of the proposed compression algorithms, we expose in the second part, the most known image quality metrics used in the evaluation of degradation of compressed images and we focus our study on our proposed method that uses textural analysis of images. Finally, in the third part, we will discuss results obtained on some chosen biometric images and validate the textural degradation in the compressed images.

\section{WAVELET-BASED IMAGE COMPRESSION}

The wavelet transform (WT) was evolved from a series of similar ideas and received a complete mathematical development. Elementary, the wavelets mathematical functions have an average value of zero and an effectively limited duration. This transform, similar to the Fourier transform, is localized in both time and frequency and make a spacio-frequency decomposition of a signal [14]. To decompose any image into wavelets coefficients require two waveforms. The first represents the low frequencies called scaling function $\Phi$ corresponding to the approximation parts of an image and the second one stands for the high frequencies called wavelet function $\Psi$, which correspond to the detailed parts. By the use of filters bank (High and Low pass), the image can be decomposed into four sub-bands namely LL (low-low band), HL (horizontal), LH (vertical), and HH (diagonal). So that, the result of multiresolution decomposition is a simultaneous image representation on various resolution levels; a low-resolution image or approximation and a higherresolution image or details, Figure 1 [15]. This will generate the hierarchical pyramidal structure.

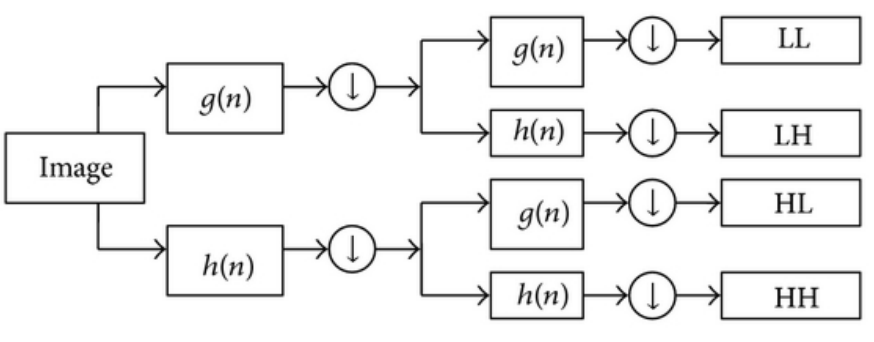

2D Wavelet decomposition

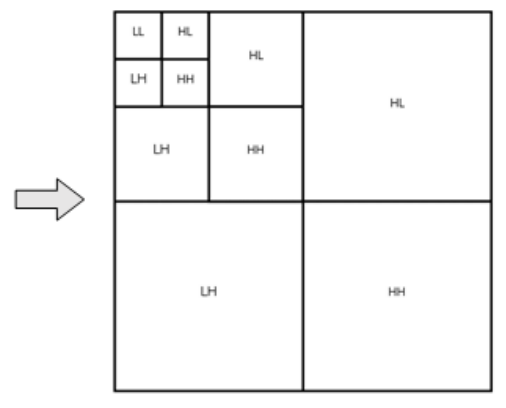

3 levels image W avelet decomposition

Figure 1. The 2D wavelet image decomposition

Swelden [16] proposed a new mathematical formulation for wavelet transformation based on the spatial construction of the biorthogonal wavelets. So that, a second-generation wavelets was proposed by [17] called the lifting-based Wavelet scheme in goal to reduce the computational complexity. The lifting-based Wavelet transform (LWT) consists of splitting phase, lifting phase, and scaling modules, treated as a prediction-error decomposition providing a complete spatial interpretation, Figure 2. There are many proposed ways to construct this lifting based code $[18,19]$. 

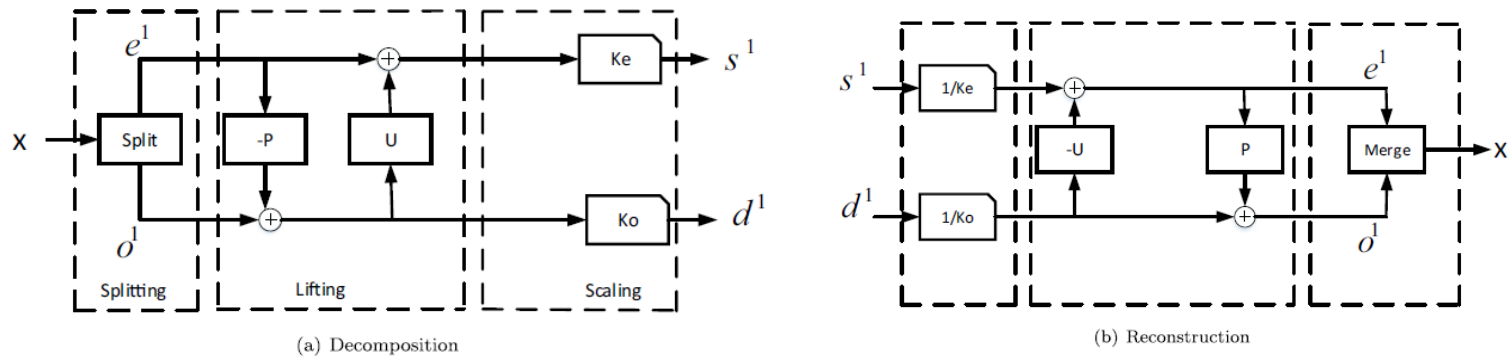

Figure 2. The lifting-based Wavelet transform decomposition and reconstruction

In other side, the use of separable dyadic analysis with classical wavelets presents a disadvantage when requiring three families of wavelets and the expansion factor between two successive scales is generally equal to 4 , which is considerably high. This decomposition manipulate a separable vertical and horizontal direction and create a diagonal representation. This has motivated scientist to create a nonseparable wavelet transforms. For this, a new analysis has been presented using only one family of wavelets with an expansion factor equal to 2 between two successive resolutions, called quincunx analysis [20, 21]. In [22], the work presents the use of quincunx wavelet compression, which suggests good results apply to the compression of medical images. In this case, the expansion matrix is equal to:

$$
M=\left[\begin{array}{rr}
1 & 1 \\
1 & -1
\end{array}\right]
$$

The transformation of the lattice is according to the following diagram [20]:

Show in Figure 3 Quincunx lattice and the corresponding Nyquist area in the frequency domain. Show in Figure 4 illustrates this type of decomposition using quincunx wavelet transform.
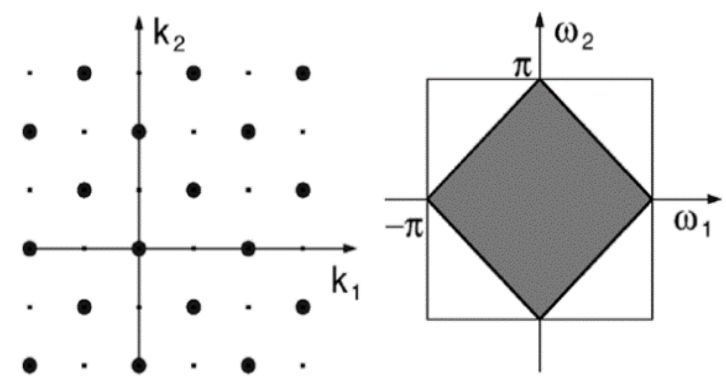

Figure 3. Quincunx lattice and the corresponding Nyquist area in the frequency domain

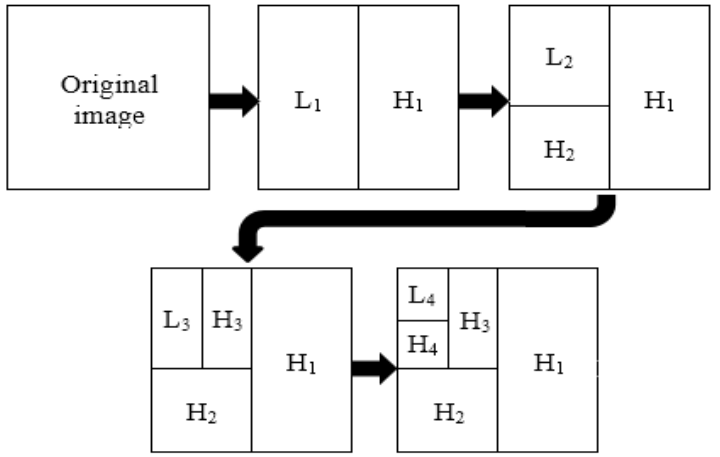

Figure 4. The Quincunx wavelet decomposition

To generate quincunx filters, we will use the standard approach consists in the applying of the diamond McClellan transform to map onto the quincunx structure [21]. However, some authors like in $[22,23]$ presented a lifting scheme for the Quincunx Wavelet transform. In our work, the algorithm model of the used quincunx image decomposition is taken from [24] and [25]. In elementary Wavelet-Based compression scheme, we couple the transform phase to an entropic encoder process. As well as traditional encoding methods such as fixed length code or variable length code, an encoding method exploit a fundamental way the tree structure of wavelet decomposition are also developed. In this case, the structure of the wavelet coefficients is considered in the form of trees and which can be coded using many wavelet-based encoding algorithms [26] like EZW, SPIHT, EPWIC, WDR and EBCOT. The interested coding algorithm in our work is the SPIHT encoder (Set Partitioning in Hierarchical Trees) which is an improved and ameliorate version of EZW algorithm proposed by Said and Pearlman [27]. In order to determine the position of the significant coefficients in the progeny of the considered coefficient, the SPIHT uses and performs a recursive partitioning of the tree, Figure 5. The success of SPIHT is due to the organization of wavelet coefficients into 
the spatial orientation trees. It is based on the creation of three list the list of significant pixels (LSP), the list of insignificant pixels (LIP) and the list of insignificant sets (LIS) with a calculated threshold T, each time a pass scan is made on both lists LSP and LIS for classifying the significant coefficient [28].

Recently, others new modified version of SPIHT was presented in literature, the most recent and important was the modified version developed by Benyahia et al. [25] and called SPIHT-Z. This version is especially adapted on the quincunx decomposition structure and it proved its performances especially in low rates. Like the original SPIHT algorithm, the SPIHT-Z considers three lists LSP, LIP and LIS with a principal modification released according to the quincunx decomposition structure of the image with a zigzag representation of the spatial tree of the SPIHT-Z, is illustrate in Figure 6. The tree dependency is organized only into the higher sub-bands by doing a zigzag scan that start from the lower high decomposition sub-band with a sweeping of all the other sub-bands.

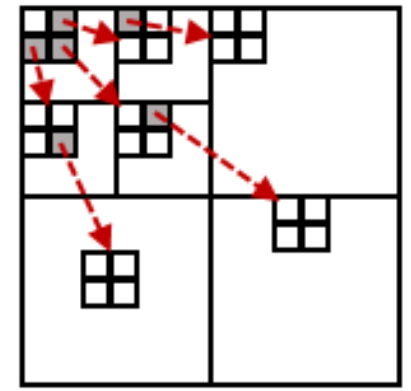

Figure 5. The repartition structure used by SPIHT algorithm

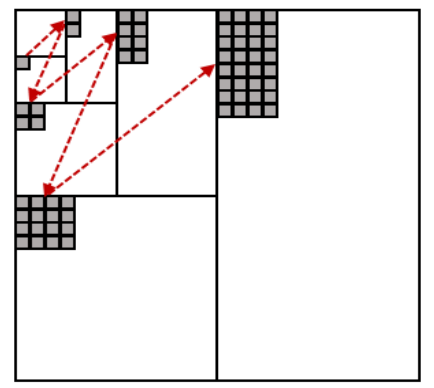

Figure 6. The coded structure used by SPIHT-Z algorithm

Using the $(n)$ decomposed $\left(H_{i}\right)$ sub-bands and starting from the $\left(H_{n}\right)$ higher sub-band of the last quincunx decomposition, the summarized SPIHT-Z algorithm is as follow:

a) If the number $i$ of the sub-band $\left(H_{i}\right)$ is even, Figure 7(a), the node pixel $(x$,$) points to two horizontal$ children pixels of the next subband $\left(H_{i-1}\right)$ with the coordinates $\left(x-L_{x}, y+L_{y}\right)$ and $\left(x-L_{x}+1, y+L_{y}\right)$.

b) If the number $i$ of the sub-band $\left(H_{i}\right)$ is an odd integer, Figure 7(b), the pixel $(x, y)$ of the sub-band $(H i)$ points to two vertical pixels of the next subband $\left(H_{i-1}\right)$ having the coordinates $\left(x+L_{x}, y-L_{y}+1\right)$ and $\left(x+L_{x}+1, y-L_{y}\right)$.

According to this pattern of scan and after an elementary zigzag cycle (even-odd scan) or (odd-even scan), each pixel will get four child descendants in the two next sub-bands, Figure 7(c).

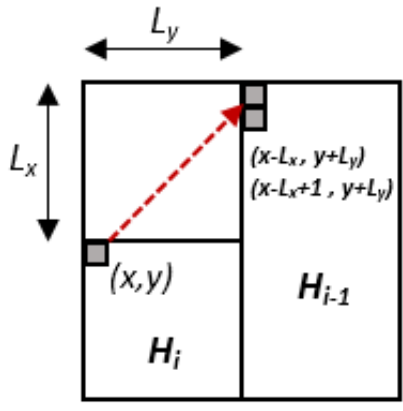

(a)

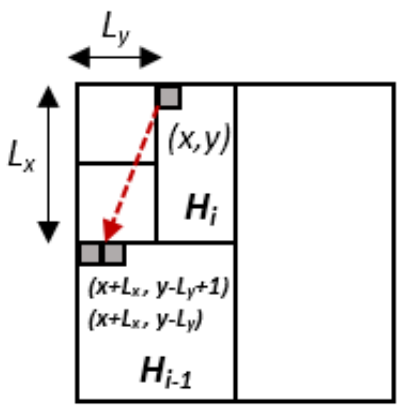

(b)

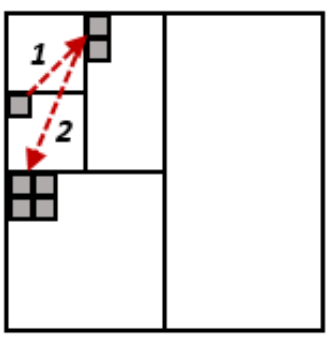

(c)

Figure 7. The SPIHT-Z algorithm pattern

In our investigation, we compare the use of the second-generation Wavelet compression scheme; the lifting Wavelet transform coupled to the SPIHT encoder (LWT-SPIHT), the lifting Quincunx Wavelet transform with the SPIHT encoder (QWT-SPIHT) and the modified SPIHT-Z encoder with lifting Quincunx Wavelet transform (QWT-SPIHTZ). 


\section{TEXTURAL IMAGE QUALITY EVALUATION}

\subsection{Image quality assessment}

Generally, to perform the used algorithms and evaluate the obtained images in compression, two ways are usable [29]; subjective methods and objective methods. In the subjective context, several measures are developed involving the human observer for assigning a note to a degraded image. These measurements respect the recommendations of ISO 3664-2000 [30] and ITU-BT.500-13 [31]. In the other context, objective methods, scientists have developed numerical image quality assessment (IQA) methods to qualify various image processing application such as image compression methods. Depending on how these quality parameters are calculated, we classify these techniques into three categories [32]; namely parameters with total reference to the original image (FR-IQA), those with reduced reference of the original image (RR-IQA) and finally those without any use of reference or no-reference (NR-IQA).

In the scientific literature, there are many image quality models starting with the simple and direct evaluation of the mean square error (MSE) between the two images, which allows the calculation of the Peak Signal to Noise Ratio (PSNR). According to our study vision, we can classify the image quality assessment (IQA) parameters into three groups: A group of Traditional evaluation parameters exploiting direct features from images. In this category and based on full reference concept, we can find the noise quality measure (NQM) index proposed by Damera-Venkata et al. [33]. The structural similarity (SSIM) index [34] considered as a milestone of the development in FR-IQA models based on the extraction of the structural information from the visual scene where some extension of SSIM are proposed also like the multi-scale SSIM (MS-SSIM) index [35]. Sheikh and Bovik [36] proposed the visual information fidelity (VIF) index, which treat the problem as an information fidelity that were quantified by the amount of information shared between the reference image and the distorted image. The Wavelet-based evaluation parameters group uses the wavelet analysis in a spatio-frequency domain of images. In the class of FR-IQA based on transforms especially the Wavelet transform. Ji and al. proposed a full-reference (FR) image quality assessment with discrete Haar wavelet transform (HWSSIM) and a multiresolution model (MRHWSSIM) [37]. Sampat et al. introduce a new measure of image similarity called the complex wavelet structural similarity (CW-SSIM) index witch is an evolution of the structural similarity index (SSIM) that tries to overcome these limitations [38]. In [39], authors propose the use of structural similarity based on discrete wavelet transform (DWTSSIM). Rezazadeh and Coulombe present another use of the wavelet structural similarity index (WSSI), more precise and less complex than the SSIM spatial index [40]. In another paper, Dumec et al. [41] presented the image quality measure based on Steerable Pyramid Wavelet Transform and structural similarity index (SSIM) called IQM2 (or SWT-SSIM). In [42], authors present a full quality assessment by using the coefficients obtained from a Haar wavelet decomposition to assess local similarities between two images, called the Haar Wavelet-Based transform Perceptual Similarity (HaarPSI) Index.

Finally, a group with texture-oriented evaluation parameters by analyzing the degradation of the textural composition of images. The first proposed method to evaluate de texture quality of image proposed by Portilla and Simoncelli [43] calculating textural statistics of complex wavelet coefficients. A structural texture similarity metric (STSIM) was proposed in [44] and another parametric quality assessment index especially for synthesized textures is presented in [45]. Zujovic [46] develop another metric for texture similarity that account for human visual perception and the stochastic nature of textures. However and in another work, a proposed method (TIQM) consists of a quality measure which qualify images in texture areas [47]. As a document image quality assessment, Alaei [48] presents an IQA based on texture similarity index (LBPSI) obtained by local LBP features. Another texture quality assessment, based on multi-scale spatial and statistical texture attributes, called (IGSTQA) index [49] using a multi-scale Spatial and statistical texture attributes of image and gradient magnitude coefficients. In our proposed investigation and to evaluation effeciently the quality degradation in the chosen biometric images, we use six parameters of the most impotant IQA metrics presented above. We use essentialy the traditional PSNR, VIF and MS-SSIM parameters. In the Wavelet-Based metrics, we choose the structural similarity based on discrete wavelet transform (DWT-SSIM) and the Haar Wavelet-Based transform perceptual similarity (HaarPSI) Index. In addition, to take part of texture-based quality evaluation, we use the (IGSTQA) index proposed by Golestaneh and Karam.

\subsection{Image texture quality}

In order to understand the influence of these compression techniques on the textual quality of compressed images, we use the Gray Level Co-Occurrence Matrix (GLCM) method that allows a statistical analysis based on selected angle and distance parameters [50]. This method estimate properties of images relating to second-order statistics measuring the probability of appearance of pairs of pixel values located at a distance in the image. This method is capable of identifying the repetition, uniformity, disorder, contrast, and heterogeneity within images. In GLCM calculation, we can specify the pixel relationships of varying direction $\theta\left(0^{\circ}, 45^{\circ}, 90^{\circ}\right.$ or $\left.135^{\circ}\right)$ and distance $\boldsymbol{d}$ from the pixel of interest, Figure 8 [51]. 


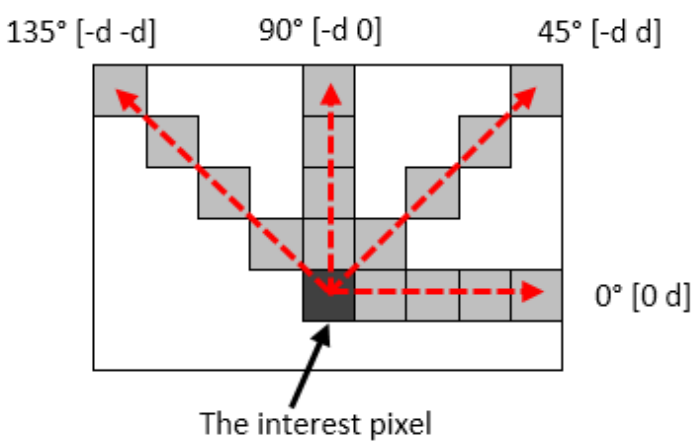

Figure 8 . The spatial relationships of pixels defined by the offsets array

The normalized GLCM matrix, interpreted as a probability mass function of the gray-level pairs in the image, is calculated by the:

$$
P=\frac{X}{\sum_{i=0}^{N-1} \sum_{j=0}^{M-1} X(i, j)}
$$

Where X represents the non-normalized GLCM calculated by counting the number of times each pair of gray-levels occurs as neighbors the $\mathrm{I}_{(\mathrm{N} \times \mathrm{M})}$ image:

$$
X(i, j)=\sum_{n=0}^{N-1} \sum_{m=0}^{M-1}\left\{\begin{array}{cc}
1 & \text { if } I(n, m)=i \text { and } I\left(n+d_{x}, m+d_{y}\right)=j \\
0 & \text { otherwise }
\end{array}\right.
$$

$\mathrm{d}_{\mathrm{x}}$ and $\mathrm{d}_{\mathrm{y}}$ are the two displacement in $\mathrm{x}$ and $\mathrm{y}$ in units of pixels.

In our case, we insvestigate in the use of five types of feature to analyse the texture features of the compressed images [50, 51]:

a) The contrast propriety measures the variance of the intensity between a pixel and its neighbors over the entire image, (3). It reflects the depth and smoothness of the image texture structure. The Contrast is equal to 0 for a constant image.

$$
\text { Contrast }=\sum_{i, j=0}^{N-1} P_{i j}(i-j)^{2}
$$

b) The correlation that reflects the similarity of the image texture in a horizontal direction or vertical direction, (4). It determines the measure of the correlation of a pixel with its neighbor. When the image is perfectly correlated its absolute value is equal to 1 (with a range in [-1 1]), while for a constant image this value is infinite.

$$
\text { Correlation }=\sum_{i, j=0}^{N-1} P_{i j} \frac{(i-\mu)(j-\mu)}{\sigma^{2}}
$$

c) The value of Energy, also known as the angular second moment, (5), determines the regularity and uniformity across the image over an interval of [0 1]. For a constant image, this value is equal to 1.

$$
\text { Energy }=\sum_{i, j=0}^{N-1}\left(P_{i j}\right)^{2}
$$

d) Homogeneity that measures the closeness of the distribution of elements to the diagonal. It allows you to configure the proximity of the distribution of GLCM elements diagonally, (6). Its value is between [0 1], with a value of 1 for a diagonal GLCM.

$$
\text { Homogeneity }=\sum_{i, j=0}^{N-1} \frac{P_{i j}}{1+(i-j)^{2}}
$$

e) The entropy parameter, which is a measure of image information and reflects the complexity of the texture distribution, (7). It measures the disorder of an image. When the image is not texturally uniform, entropy is very large. 


$$
\text { Entropy }=\sum_{i, j=0}^{N-1}-\ln \left(P_{i j}\right) P_{i j}
$$

To perform degradation in texture of the compressed image, we calculate for each cited textural features (Contrast, Correlation, Energy, Entropy or Homogeneity) a difference compared to the original image as in (8):

$$
\Delta F=\frac{\left|F_{\text {Compressed }}-F_{\text {Original }}\right|}{F_{\text {Original }}}
$$

In addition to normalization with respect to the unit, we use the following equation for calculating the image texture quality for each textural feature using one of:

$$
\text { ITQ } Q_{\text {Feature }}=1-\Delta F
$$

Finally, we define our proposed global image texture quality (ITQ) as a following textural features:

$$
I T Q=\frac{I T Q_{\text {Contrast }}+I T Q_{\text {Correlation }}+I T Q_{\text {Energy }}+I T Q_{\text {Homogeneity }}+I T Q_{\text {Entropy }}}{5}
$$

We note that Haralick texture features are common texture descriptors in image analysis. To calculate these features, the image gray-levels are reduced using a quantization process that make results depending heavily on the quantization step. An important and recent work [52] developed a modified Haralick features that are invariant to the number of quantization gray-levels. In this method, the interpretation of the GLCM as a discrete approximation of a probability density function, which remedies the disadvantage of the sensitivity of the textures to the chosen quantification in the classical GLCM method.

\section{RESULTS AND DISCUSSION}

In this article, we give numerical experiments to test the effect of compression on some biometric images. We choose the use of two kinds of images:

a) The first biometric image is an iris gray image from the IIT Delhi Iris database at Biometrics Research Laboratory in IIT Delhi, New Delhi, India [53] with 320x240 pixels and 8 bpp resolution, Figure 9(a),

b) The second biometric image represents a 8 bit gray level palmprint image taken from the CASIA Palmprint Database collection realized by the Chinese Academy of Sciences Institute of Automation (CASIA) with 640x480 pixels [54], Figure 9(b).

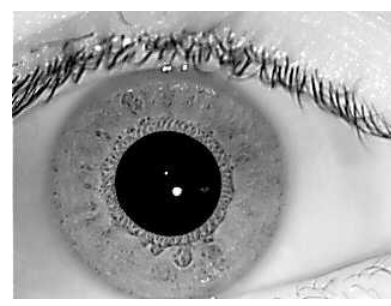

(a)

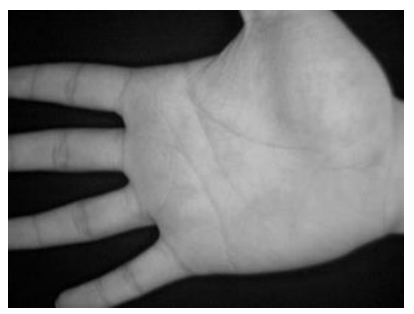

(b)

Figure 9. The original biometrics images used in our investigation

The evaluation of the two biometric images are presented in Figures 10 to 15 for the iris and the Palmprint images. These curves plotted against the compression ratio (in bpp), show the variation of the standard quality indices PSNR, VIF and MS-SSIM as well as some specific indices like DWT-SSIM, HaarPSI and IGSTQA. We can observe clearly that the best results are improved by the Quincunx Wavelet compression using the SPIHT-Z encoder, especially in low rates (under $1.0 \mathrm{bpp}$ ). Considering the PSNR evaluation, the quincunx wavelet compression with SPIHT-Z is more adapted with the palmprint image than in iris image especilly in low bitrates. In the case of palmprint, this algorithm is satisfying from a low compression rate $<0.1 \mathrm{bpp}$, however, in iris image from $0.2 \mathrm{bpp}$. The same remarques are clearly seen in the others metrics curves in low bitrates part (under $0.2 \mathrm{bpp}$ ). This can be explain by the textural components higher in iris image than in plamprint as shown in Table 1. 

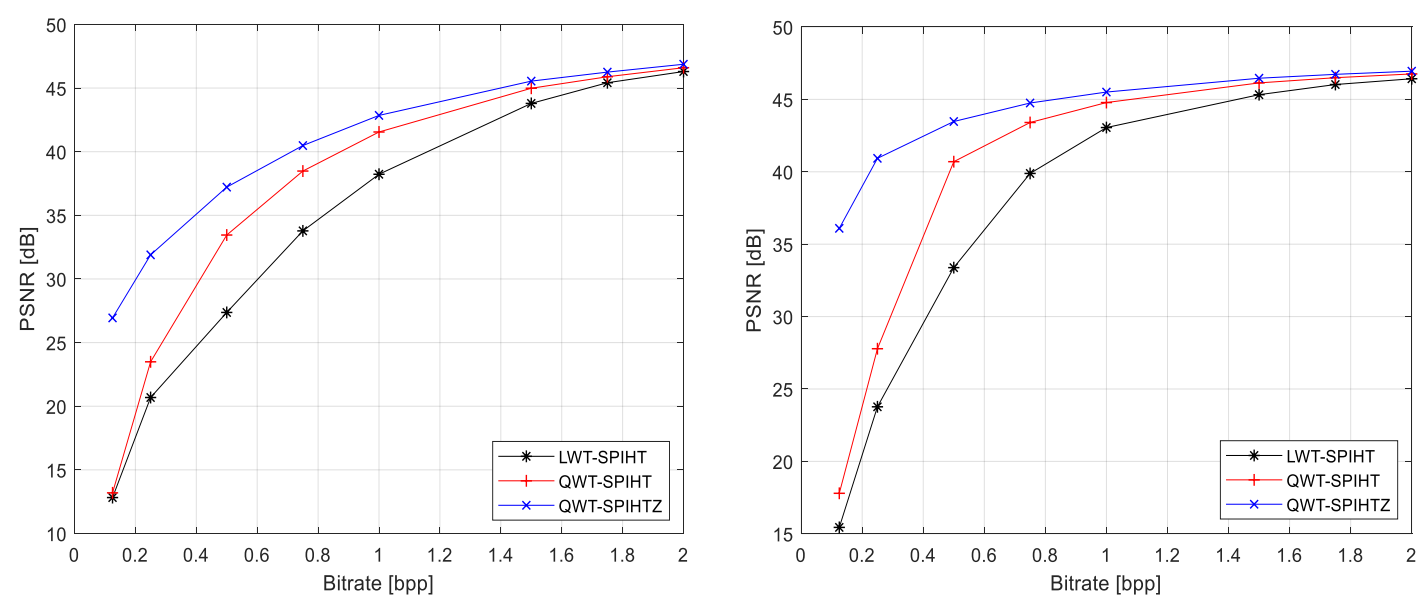

Figure 10. The variation of the PSNR in the Iris image (left) and Palmprint image (right)
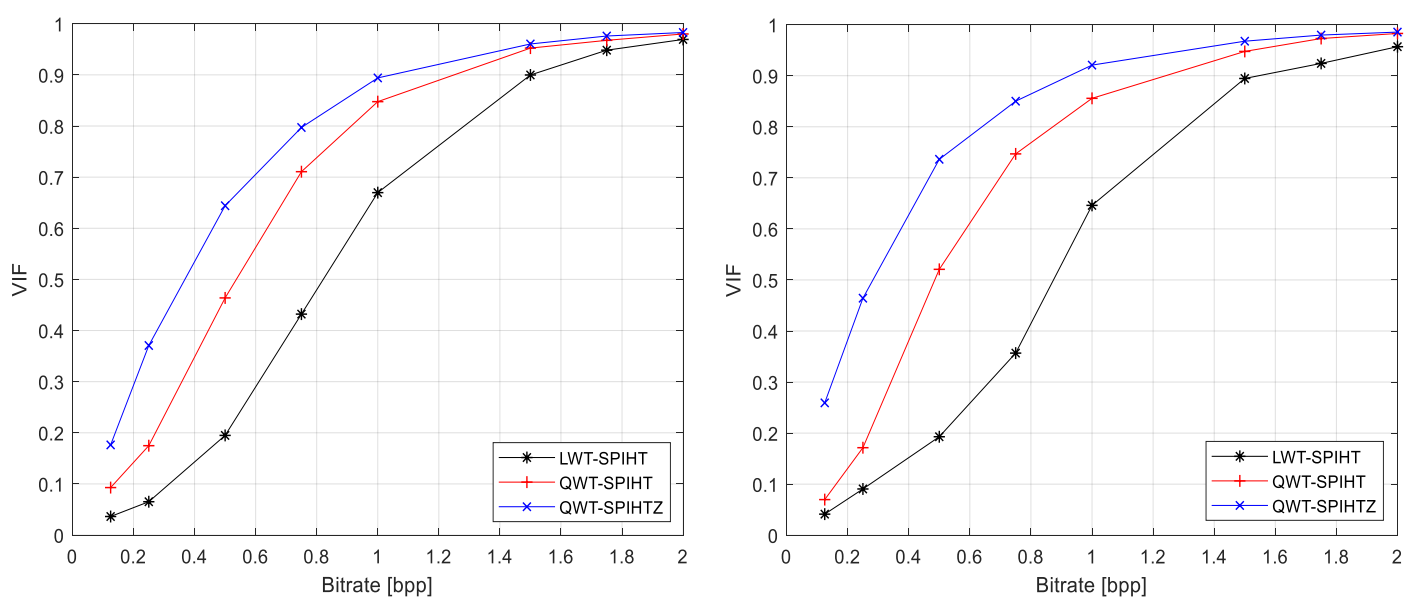

Figure 11. The variation of the VIF in the Iris image (left) and Palmprint image (right)
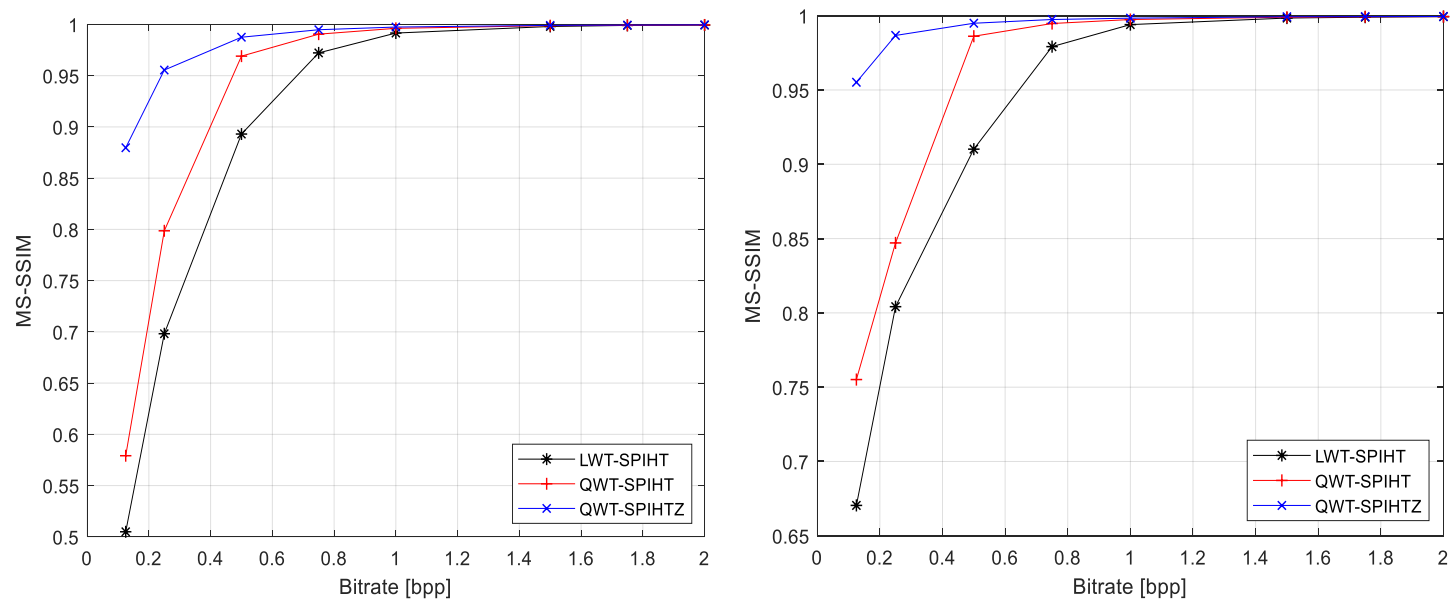

Figure 12. The variation of the MS-SSIM in the Iris image (left) and Palmprint image (right) 

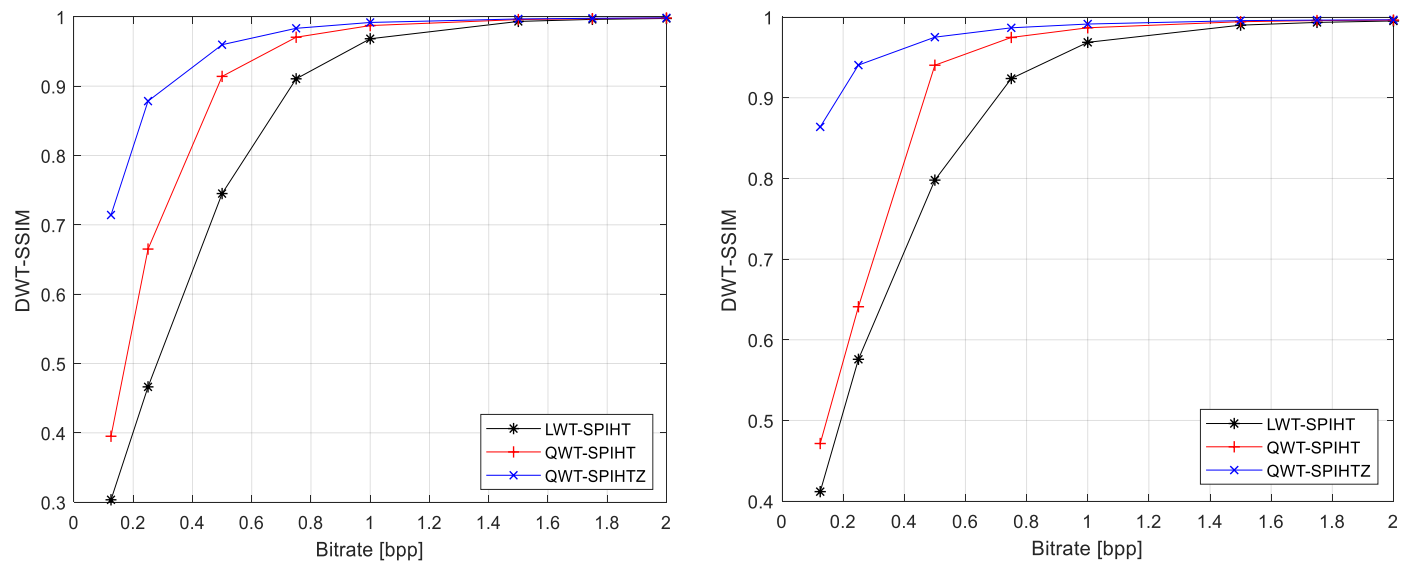

Figure 13. The variation of the DWT-SSIM in the Iris image (left) and Palmprint image (right)
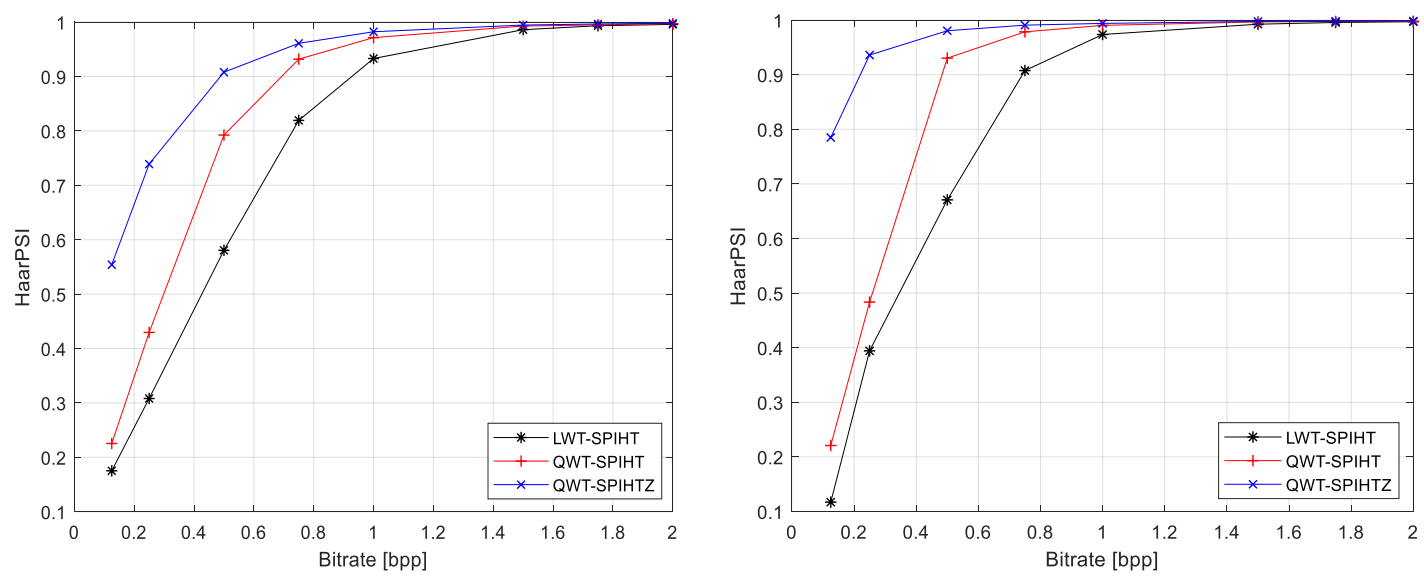

Figure 14. The variation of the HaarPSI in the Iris image (left) and Palmprint image (right)
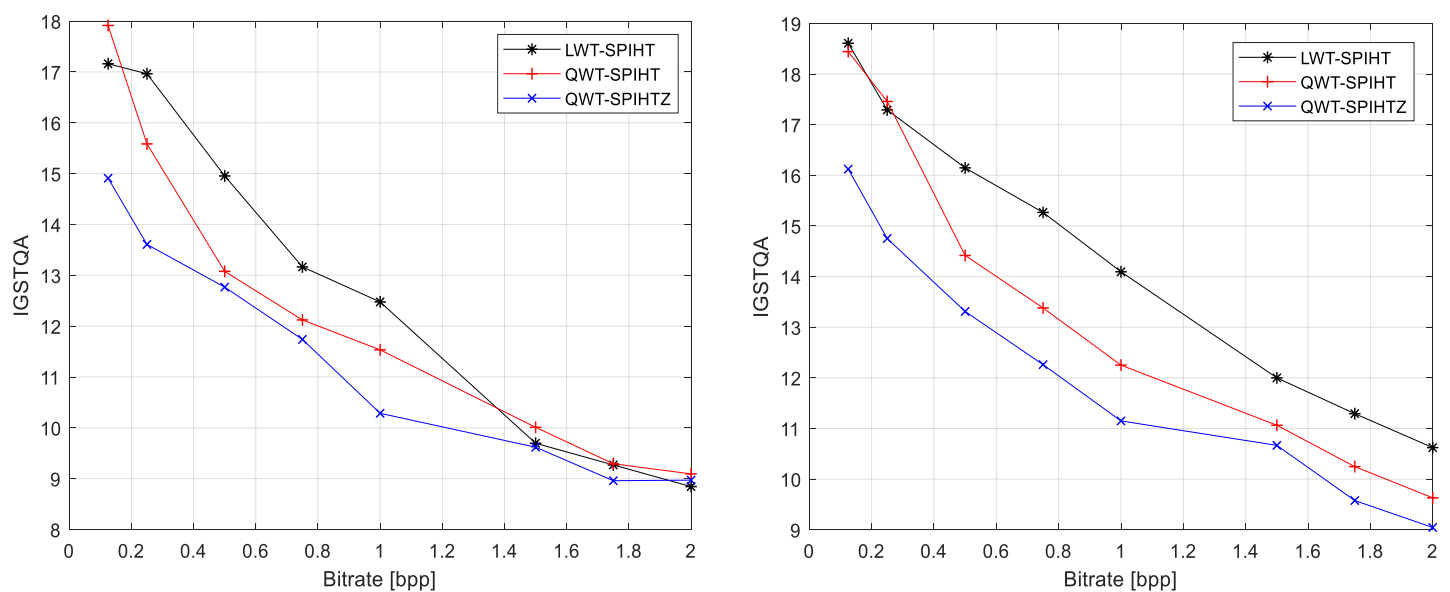

Figure 15. The variation of the IGSTQA in the Iris image (left) and Palmprint image (right)

The classical GLCM parameters calculated for the original images, are shown in Table 1, show that the iris image is more textured than that of the original palmprint image. It has high contrast and low energy, while the palmprint image is more correlated and homogeneous. 
Table 1. The GLCM textural characteristics of original images

\begin{tabular}{lllllll}
\hline \multirow{4}{*}{ IRIS } & CONT & CORR & ENER & ENTR & HOMO \\
\hline & $\theta=0^{\circ}$ & 0.2432 & 0.9675 & 0.1216 & 2.4605 & 0.9156 \\
& $\theta=45^{\circ}$ & 0.2715 & 0.9637 & 0.1169 & 2.5062 & 0.9066 \\
& $\theta=135^{\circ}$ & 0.1197 & 0.9840 & 0.1334 & 2.2967 & 0.9439 \\
& Mean & 0.2386 & 0.9572 & 0.1143 & 2.5457 & 0.8989 \\
& Invariant & 0.2603 & 0.9788 & 0.4677 & 2.7236 & 0.9975 \\
& $\theta=0^{\circ}$ & 0.0321 & 0.9964 & 0.2523 & 1.6146 & 0.9840 \\
& $\theta=45^{\circ}$ & 0.0469 & 0.9948 & 0.2487 & 1.6585 & 0.9768 \\
& $\theta=90^{\circ}$ & 0.0375 & 0.9958 & 0.2525 & 1.6253 & 0.9812 \\
& $\theta=135^{\circ}$ & 0.0526 & 0.9941 & 0.2478 & 1.6717 & 0.9738 \\
& Mean & 0.0423 & 0.9953 & 0.2504 & 1.6426 & 0.9789 \\
& Invariant & 0.0213 & 0.9985 & 0.8519 & 3.8474 & 0.9997 \\
\hline
\end{tabular}

The effect of the compression process on the texture structure of the two biometric images are calculated and shown in the Figures 16 to 20 with comparison of LWT-SPIHT, QWT-SPIHT, QWT-SPIHTZ and JPEG2000 algorithms. We can note clearly, that the compression with Quincunx Wavelet with the SPIHT$\mathrm{Z}$ preserves the textual quality of these images almost like that with the JPEG2000 compression algorithm.
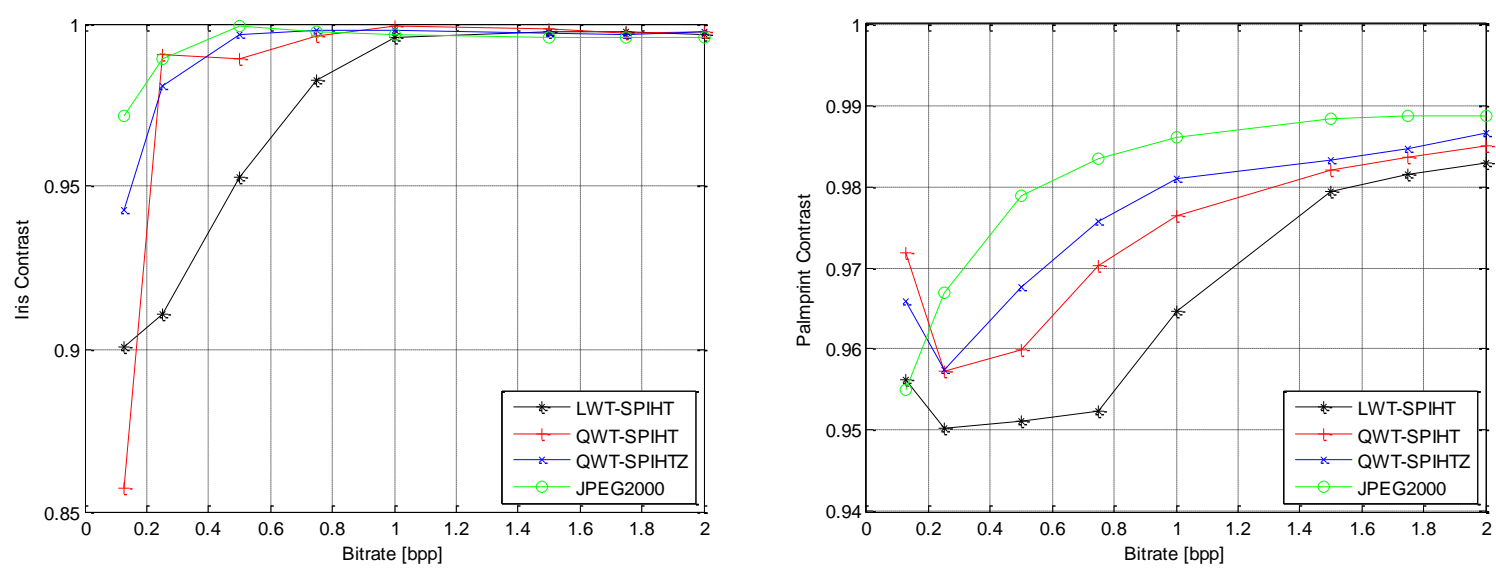

Figure 16. The image contrast quality for Iris (left) and Palmprint (right) images
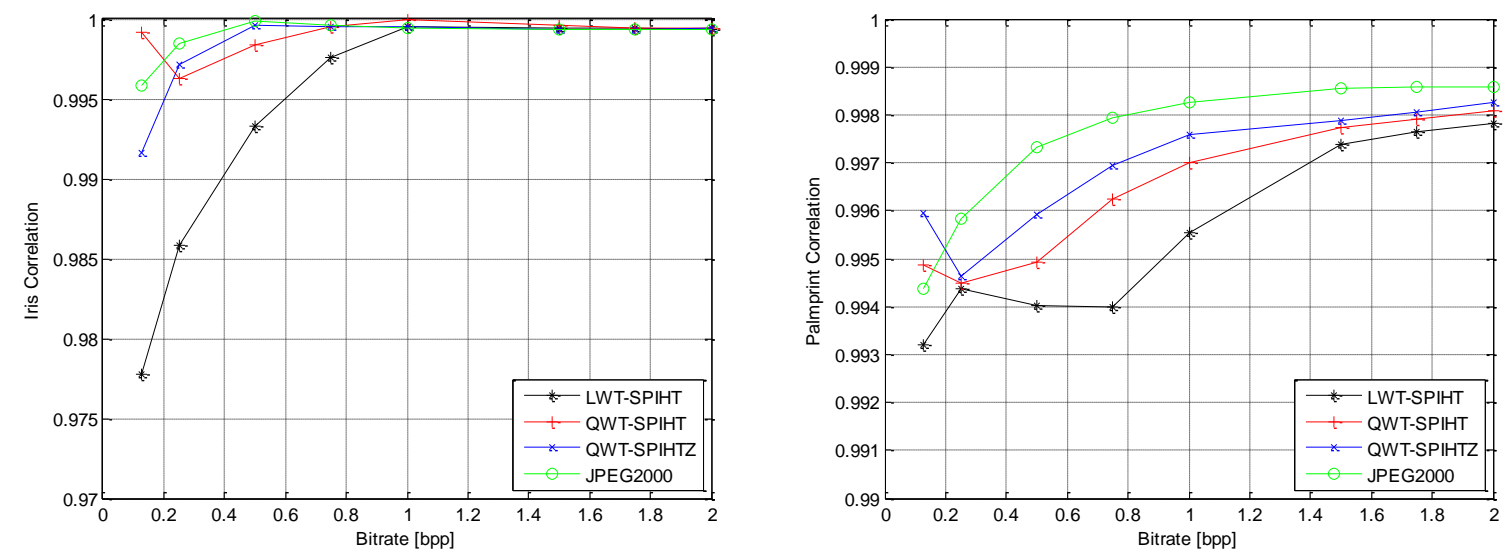

Figure 17. The image correlation quality for Iris (left) and Palmprint (right) images 

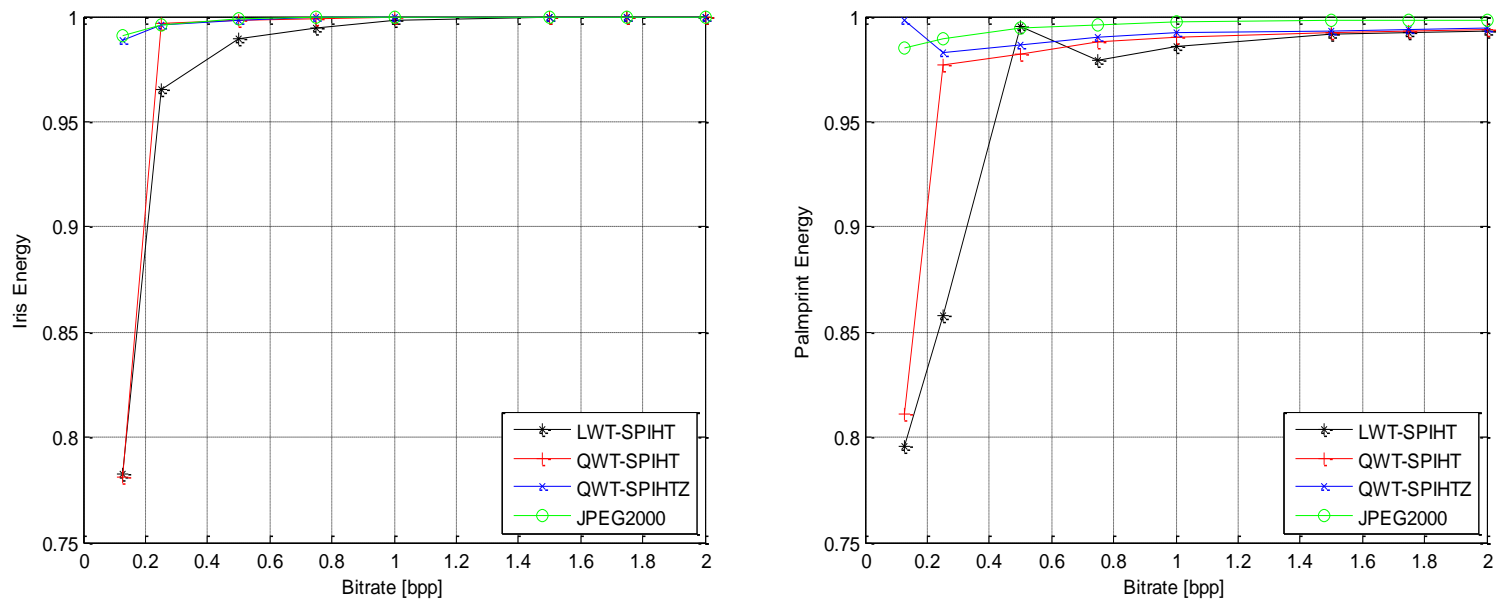

Figure 18. The image Energy quality for Iris (left) and Palmprint (right) images
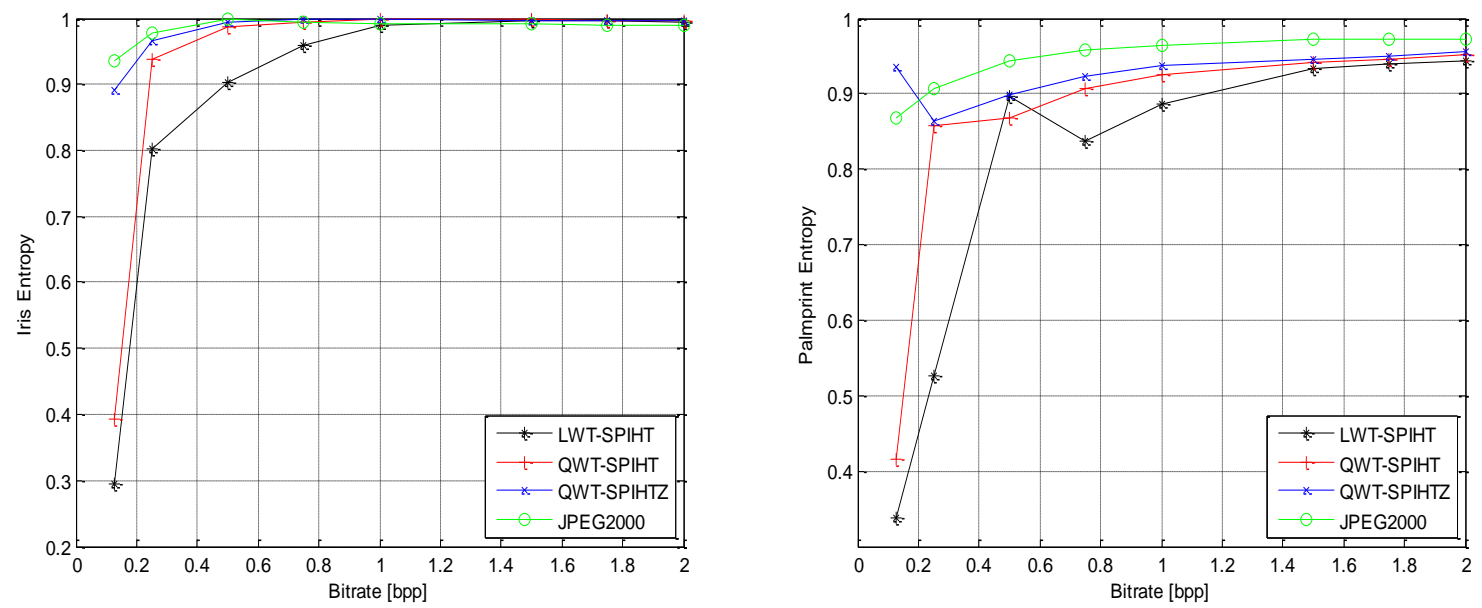

Figure 19. The image entropy quality for Iris (left) and Palmprint (right) images
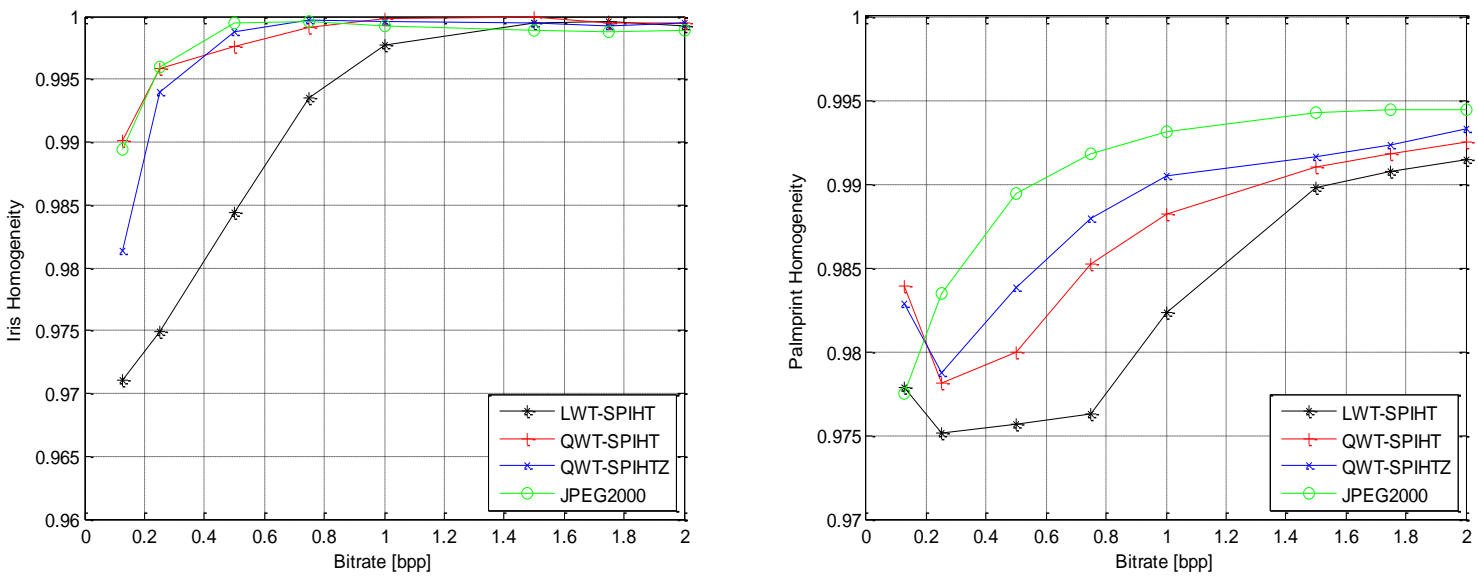

Figure 20. The image homogeneity quality for Iris (left) and Palmprint (right) images 
Finally, we can present the variation of our proposed image texture quality according to the selected compression algorithms and note the efficiency of the QWT-SPIHTZ and JPEG2000 compression algorithms in the preservation of the textural quality of biometric images especially in low bitrates, Figure 21.
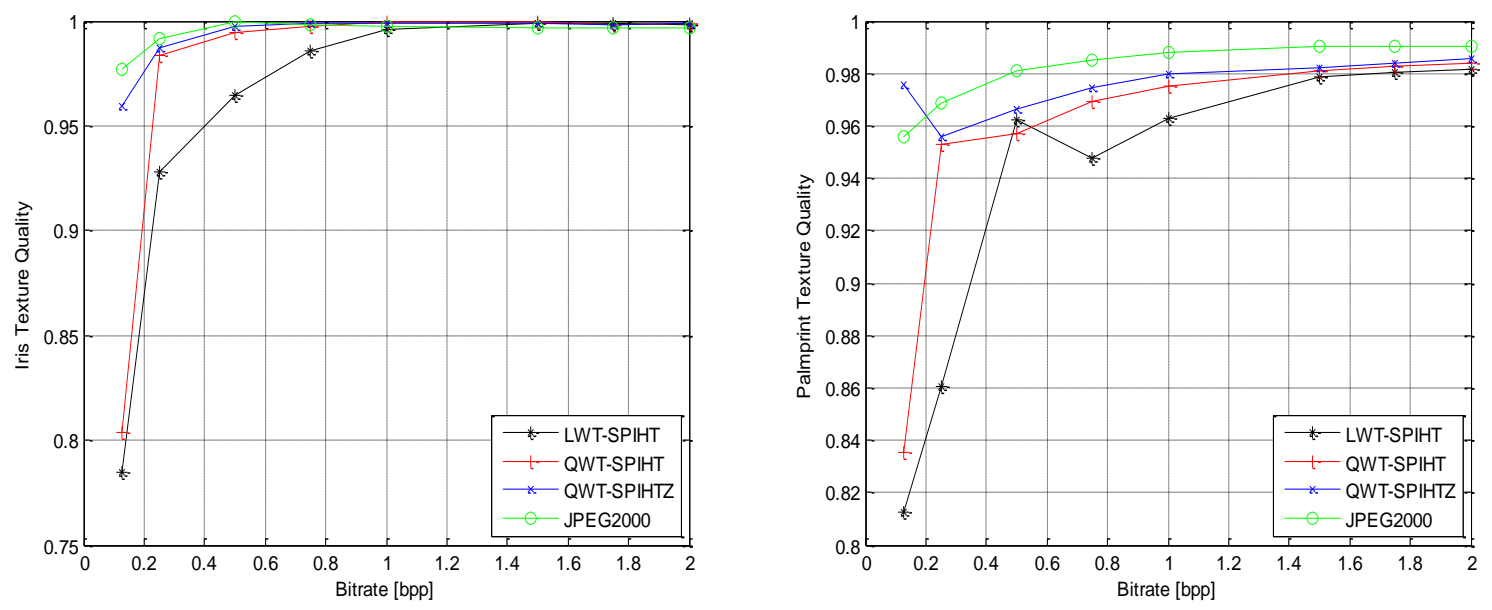

Figure 21. The variation of image textural quality in Iris (left) and Palmprint (right) images.

We can conclude that the compression technique with Quincunx Wavelet transform associate to the new SPIHT-Z encoder like JPEG2000 presents a reliable quality in the case of biometric images, especially in low bitrates, which preserves the textual quality of these images.

\section{CONCLUSION}

In this paper, we presented results for a comparative study of some second-generation wavelet compression coupled with SPIHT and SPIHT-Z coding on biometric images. To evaluate and qualify the efficacy of these algorithms, we used full reference classical image quality assessment, Wavelet-based image quality assessment and Texture-oriented image quality assessment. In this work we perform a textural analysis by calculating an image textural quality using the elementary GLCM texture features like Contrast, Correlation, Energy, Entropy or Homogeneity. These results confirmed that the Quincunx based algorithm associated to SPIHT-Z (QWT-SPIHTZ) like JPEG2000 gives good results than others used algorithms (LWT-SPIHT and QWT-SPIHT). It is worth mentioning that at very low bitrates, these algorithms provide very important textural quality in the biometric images.

\section{ACKNOWLEDGEMENTS}

This work is partly supported by Algerian ministry of higher education and research (PRFU) project $\mathrm{N}^{\circ}$ A25N01UN080120180002.

\section{REFERENCES}

[1] R. C. Gonzalez and R. E. Woods, Digital image processing. New York, NY: Pearson, 2007. ISBN: 9780131687288

[2] ISO/IEC 10918, "Information technology: Digital compression and coding of continuous-tone still images: Requirements and guidelines," ISO/IEC, 1994.

[3] ISO/IEC 15444, "Information technology - JPEG 2000 image coding system: Core coding system," ISO/IEC, 2016.

[4] ISO/IEC 19794, "Information technology: Biometric data interchange formats", ISO/IEC, 2011.

[5] R. C. Kidd, "Comparison of wavelet scalar quantization and JPEG for fingerprint image compression," J. Electron. Imaging, vol. 4, no. 1, pp. 31, Jan. 1995.

[6] G. A. Khuwaja, "Best parameter based compression of fingerprints with wavelet packets," International Journal of Computer Applications in Technology (IJCAT), vol. 19, no. 1, pp. 51-56, 2004.

[7] W. Funk, M. Arnold, C. Busch, and A. Munde, "Evaluation of image compression algorithms for fingerprint and face recognition systems," in Proceedings from the Sixth Annual IEEE Systems, Man and Cybernetics (SMC) Information Assurance Workshop, 2005., West Point, NY, USA, pp. 72-78, 2005. 
[8] A. Mascher-Kampfer, H. Stögner, and A. Uhl, "Comparison of compression algorithms impact on fingerprint and face recognition accuracy," in the Electronic Imaging 2007, San Jose, CA, USA, pp. 650810, 2007.

[9] R. W. Ives, D. A. Bishop, Y. Du, and C. Belcher, "Iris Recognition: the consequences of image compression," EURASIP J. Adv. Signal Process., vol. 2010, no. 1, pp. 680845, Dec. 2010.

[10] A. Paul, T. Z. Khan, P. Podder, R. Ahmed, M. M. Rahman, and M. H. Khan, "Iris image compression using wavelets transform coding," in 2015 2nd International Conference on Signal Processing and Integrated Networks (SPIN), Noida, Delhi-NCR, India, pp. 544-548, 2015.

[11] C. Rathgeb, A. Pflug, J. Wagner, and C. Busch, "Effects of image compression on ear biometrics," IET Biometrics, vol. 5, no. 3, pp. 252-261, Sep. 2016.

[12] V. Ablinger, C. Zenz, J. Hammerle-Uhl, and A. Uhl, "Compression standards in finger vein recognition," in 2016 International Conference on Biometrics (ICB), Halmstad, Sweden, pp. 1-7, 2016.

[13] V. A. Anju and S. Priya, "A Study on Different Types of FR IQA Classification", Intern. J. of Innov. Res. in Science, Engin. and Techn., vol. 6, no 5, p. 46-51, Mars 2017.

[14] M. Antonini, M. Barlaud, P. Mathieu, and I. Daubechies, "Image coding using wavelet transform," IEEE Transactions on Image Processing, vol. 1, no. 2, pp. 205-220, Apr. 1992.

[15] R. Boujelbene, Y. B. Jemaa, and M. Zribi, "A comparative study of recent improvements in wavelet-based image coding schemes", Multimed Tools Appl, vol. 78, no. 2, pp. 1649-1683, Jan. 2019.

[16] W. Sweldens, "The Lifting Scheme: A Custom-Design Construction of Biorthogonal Wavelets," Applied and Computational Harmonic Analysis, vol. 3, no. 2, pp. 186-200, Apr. 1996.

[17] I. Daubechies and W. Sweldens, "Factoring wavelet transforms into lifting steps," The Journal of Fourier Analysis and Applications, vol. 4, no. 3, pp. 247-269, May 1998.

[18] I. Boukli-Hacene, M. Beladghem, and A. Bessaid, "Lossy Compression Color Medical Image Using CDF Wavelet Lifting Scheme," International Journal of Image, Graphics and Signal Processing (IJIGSP), vol. 5, no. 11, pp. 5360, Sep. 2013.

[19] T. Taha, P. Ehkan, and R. Ngadiran, "A new perceptual mapping model using lifting wavelet transform," MATEC Web Conf., vol. 140, pp. 01036, 2017.

[20] D. Van De Ville, T. Blu, and M. Unser, "On the multidimensional extension of the quincunx subsampling matrix," IEEE Signal Process. Lett., vol. 12, no. 2, pp. 112-115, Feb. 2005.

[21] Y. Chen, M. D. Adams, and W.-S. Lu, "Design of Optimal Quincunx Filter Banks for Image Coding," EURASIP J. Adv. Signal Process., vol. 2007, no. 1, pp. 083858, Dec. 2006.

[22] A. Gouze, M. Antonini, and M. Barlaud, "Quincunx lifting scheme for lossy image compression," in Proceedings 2000 International Conference on Image Processing (Cat. No.00CH37101), Vancouver, Canada, vol. 1, pp. 665668, 2002.

[23] M. H. Jansen and P. J. Oonincx, "Second generation wavelets and applications," Springer-Verlag London, 2005.

[24] M. Beladgham, A. Bessaid, A. Taleb-Ahmed, and I. Boucli-Hacene, "Medical image compression using quincunx wavelets and SPIHT coding," Journal of Electrical Engineering and Technology, vol. 7, no. 2, pp. 264-272, 2012.

[25] I. Benyahia, A. Bassou, C. Allaoui, M. Beladgham, "Modified spiht algorithm for quincunx wavelet image coding," Indonesian Journal of Electrical Engineering and Computer Science (IJEECS), vol. 16, no. 1, pp. 230-242, 2019.

[26] V. J. Rehna, "Wavelet based image coding schemes: A recent survey", International Journal on Soft Computing (IJSC), vol. 3, no. 3, pp. 101-118, Aug. 2012.

[27] A. Said and W. A. Pearlman, "A new fast and efficient image codec based on set partitioning in hierarchical trees," IEEE Transactions on Circuits and Systems for Video Technology, vol. 6, no. 3, pp. 243-250, 1996.

[28] I. Benyahia, M. Beladgham, and A. Bassou, "Evaluation of the medical image compression using wavelet packet transform and SPIHT coding," International Journal of Electrical and Computer Engineering (IJECE), vol. 8, no. 4, pp. 2139-2147, Aug. 2018.

[29] B. S. Phadikar, G. K. Maity, and A. Phadikar, "Full Reference Image Quality Assessment: A Survey," in Industry Interactive Innovations in Science, Engineering and Technology, vol. 11, Eds. Singapore: Springer Singapore, pp. 197-208, 2018.

[30] ISO 3664, "Viewing Conditions for Graphic Technology and Photography," Revised by ISO 3664:2009. ISO, 2000.

[31] ITU-BT.500-13, "Methodology for the subjective assessment of the quality of television pictures". ITU, 2012.

[32] L. Zhang, L. Zhang, X. Mou, and D. Zhang, "A comprehensive evaluation of full reference image quality assessment algorithms," in 2012 19th IEEE International Conference on Image Processing, Orlando, FL, USA, pp. 1477-1480, 2012.

[33] N. Damera-Venkata, T. D. Kite, W. S. Geisler, B. L. Evans, and A. C. Bovik," Image quality assessment based on a degradation model", IEEE Transactions on Image Processing, vol. 9, no. 4, pp. 636-650, Apr. 2000.

[34] Z. Wang, A. C. Bovik, H. R. Sheikh, and E. P. Simoncelli, "Image Quality Assessment: From Error Visibility to Structural Similarity," IEEE Trans. on Image Process., vol. 13, no. 4, pp. 600-612, Apr. 2004.

[35] Z. Wang, E. P. Simoncelli, and A. C. Bovik, "Multiscale structural similarity for image quality assessment," in The Thrity-Seventh Asilomar Conference on Signals, Systems \& Computers, Pacific Grove, CA, USA, 2003, pp. 1398-1402, 2003.

[36] H. R. Sheikh and A. C. Bovik, "Image information and visual quality," IEEE Trans. on Image Process., vol. 15, no. 2, pp. 430-444, Feb. 2006

[37] G. L. Ji, X. M. Ni, and H. Y. Bae, "A full-reference image quality assessment algorithm based on haar wavelet transform," in 2008 International Conference on Computer Science and Software Engineering, Wuhan, China, pp. 791-794, 2008. 
[38] M. P. Sampat, Z. Wang, S. Gupta, A. C. Bovik, and M. K. Markey, "Complex wavelet structural similarity: A new image similarity index," IEEE Transactions on Image Processing, vol. 18, no. 11, pp. 2385-2401, Nov. 2009.

[39] C. Yang, W. Gao, L. Po, "Discrete wavelet transform-based structural similarity for image quality assessment," in 2008 15th IEEE International Conference on Image Processing, pp. 377-380, 2008.

[40] S. Rezazadeh and S. Coulombe, "A novel discrete wavelet transform framework for full reference image quality assessment," Signal, Image and Video Processing (SIViP), vol. 7, no 3, pp. 559-573, May 2013.

[41] E. Dumic, S. Grgic, and M. Grgic, "IQM2: new image quality measure based on steerable pyramid wavelet transform and structural similarity index," Signal, Image and Video Processing (SIViP), vol. 8, no 6, pp. 1159-1168, Sep. 2014.

[42] R. Reisenhofer, S. Bosse, G. Kutyniok, and T. Wiegand, "A haar wavelet-based perceptual similarity index for image quality assessment," Signal Processing: Image Communication, vol. 61, pp. 33-43, Feb. 2018.

[43] J. Portilla and E. P. Simoncelli, "A parametric texture model based on joint statistics of complex wavelet coefficients," International Journal of Computer Vision, vol. 40, no 1, pp. 49-70, 2000.

[44] X. Zhao, M. G. Reyes, T. N. Pappas, and D. L. Neuhoff, "Structural texture similarity metrics for retrieval applications," In IEEE International Conference on Image Processing (ICIP), San Diego, USA, pp. 1196-1199, 12-15 Oct. 2008.

[45] D. S. Swamy, K. J. Butler, D. M. Chandler, and S. S. Hemami, "Parametric quality assessment of synthesized textures," In IS\&T/SPIE Electronic Imaging, pp. 78650B-78658B, 2011.

[46] J. Zujovic, T. N. Pappas, and D. L. Neuhoff, "Structural texture similarity metrics for image analysis and retrieval," IEEE Transactions on Image Processing, vol. 22, no 7, pp. 2545-2558, 2013.

[47] S. Maksimovic-Moicevic, Z. Lukac, and M. Temerinac, "Edge-texture 2D image quality metrics suitable for evaluation of image interpolation algorithms," Computer Science and Information Systems (ComSIS), vol. 12, no. 2, pp. 405-425, 2015.

[48] A. Alaei, D. Conte, M. Blumenstein, and R. Raveaux, "Document image quality assessment based on texture similarity index," in 2016 12th IAPR Workshop on Document Analysis Systems (DAS), pp. 132-137, 2016.

[49] S. A. Golestaneh and L. J. Karam, "Synthesized texture quality assessment via multi-scale spatial and statistical texture attributes of image and gradient magnitude coefficients," In Proceedings of the IEEE Conference on Computer Vision and Pattern Recognition Workshops, Salt Lake City, UT, USA, 18-22 June 2018.

[50] R. M. Haralick, K. Shanmugam, et I. Dinstein, "Textural features for image classification," IEEE Trans. Syst., Man, Cybern., vol. SMC-3, no 6, pp. 610-621, Nov. 1973.

[51] P. Mohanaiah, P. Sathyanarayana and L. GuruKumar, "Image texture feature extraction using GLCM approach," International Journal of Scientific and Research Publications (IJSRP), vol. 3, no 5, pp. 1-5, May 2013.

[52] T. Löfstedt, P. Brynolfsson, T. Asklund, T. Nyholm, and A. Garpebring, "Gray-level invariant Haralick texture features," PLoS ONE, vol. 14, no 2, pp. e0212110, Feb. 2019.

[53] The IIT Delhi Iris Database. Available at: http://www4.comp.polyu.edu.hk/ csajaykr/IITD/Database_Iris.htm

[54] The Casia Palmprint Image Database. Available at: http://biometrics.idealtest.org/dbDetailForUser.do?id=5

\section{BIOGRAPHIES OF AUTHORS}

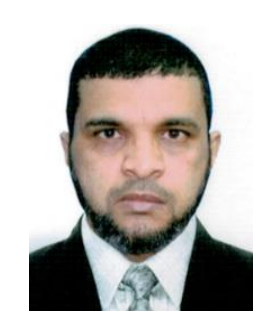

Ahmed Bouida was born in Bechar (Algeria), received Bachelor in 1989 and Engineers degree in Electrical Engineering from the University Djillali LIABES of Sidi Bel Abbes (Algeria) in 1994 and his Magister degree in Electronic from the University Djillali LIABES of Sidi Bel Abbes (Algeria) in 1998. His current research interest includes Image processing, Biometric image compression, Second generation wavelets transform and Image quality assessment. Email: ahmedbouida@yahoo.fr

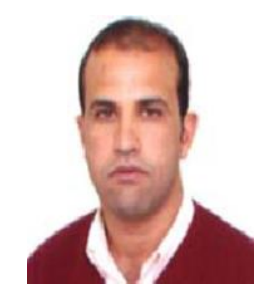

Mohammed Beladgham was born in Tlemcen, Algeria. He received the electrical engineering diploma from university of Tlemcen, Algeria, and then a Master in signals and systems from University of Tlemcen, Algeria and the $\mathrm{PhD}$ degree in Electronics from the University of Tlemcen (Algeria), in 2012. He was an Associate Professor at the University of Bechar, Algeria. Since 2015. He is currently a Professor at University of Bechar in the department of Electical Engineering, and does his research at the LTIT Laboratory, Tahri Mohammed University Bechar. His research interests are Image and video processing, Image segmentation Medical image compression, Biomedical imaging, Biometric systems, wavelets transform and optimal encoder. Email: beladgham.tlm@gmail.com

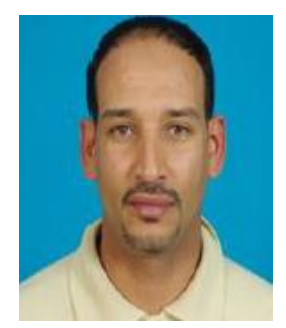

Abdesselam Bassou was born in Bechar, Algeria. He received the Dipl.El.-Ing. Degree from the University of Tlemcen, Algeria in 1997, his Master from the University of Sidi Bel Abbes, Algeria in 2000, and his Doctoral degree Es Science from the University of Sidi Bel Abbes, Algeria in 2006. Actually, $\mathrm{He}$ is an Associate Professor at University of Bechar, Algeria. His main interests are digital signal processing, turbo encoding schemes and iterative decoding over fading channels, and channel equalization Correspondance address: Information Processing and Telecommunication Laboratory (LTIT), Tahri Mohammed University, Bechar 08000, Algeria. Email: a.bassou@gmail.com 


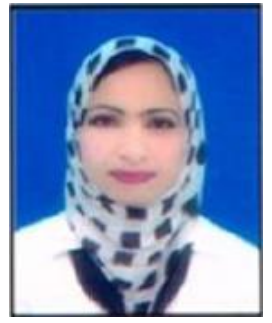

Ismahane Benyahia was born in Bechar, Algeria. She received the dipl. El-Ing from the University of Bechar University, Algeria, in 2009, the Master degree in signals and telecommunication from University of Bechar, Algeria in 2014, and her Doctoral degree Es Science from the University of Bechar, Algeria. Here main interested are Image and video processing, $1 \mathrm{G}$ and $2 \mathrm{G}$ wavelets transform. Correspondance address: Information Processing and Telecommunication Laboratory (LTIT), Tahri Mohammed University, Bechar 08000, Algeria. E-mail: ismahanelv4@gmail.com 